\title{
A Semantic Ontology for Disaster Trail Management System
}

\author{
Ashfaq Ahmad ${ }^{1}$, Roslina Othman ${ }^{2}$, Mohamad Fauzan $^{3}$, Qazi Mudassar Ilyas ${ }^{4}$ \\ Kulliyyah of Information and Communication Technology \\ International Islamic University Malaysia, Kuala Lumpur, Malaysia ${ }^{1,2,3}$ \\ College of Computer Science \& Information Technology, Jazan University, Jazan, Saudi Arabia ${ }^{1}$ \\ College of Computer Sciences and Information Technology, King Faisal University, Saudi Arabia ${ }^{4}$
}

\begin{abstract}
Disasters, whether natural or human-made, leave a lasting impact on human lives and require mitigation measures. In the past, millions of human beings lost their lives and properties in disasters. Information and Communication Technology provides many solutions. The issue of so far developed disaster management systems is their inefficiency in semantics that causes failure in producing dynamic inferences. Here comes the role of semantic web technology that helps to retrieve useful information. Semantic web-based intelligent and self-administered framework utilizes XML, RDF, and ontologies for a semantic presentation of data. The ontology establishes fundamental rules for data searching from the unstructured world, i.e., the World Wide Web. Afterward, these rules are utilized for data extraction and reasoning purposes. Many disaster-related ontologies have been studied; however, none conceptualizes the domain comprehensively. Some of the domain ontologies intend for the precise end goal like the disaster plans. Others have been developed for the emergency operation center or the recognition and characterization of the objects in a calamity scene. A few ontologies depend on upper ontologies that are excessively abstract and are exceptionally difficult to grasp by the individuals who are not conversant with theories of the upper ontologies. The present developed semantic web-based disaster trail management ontology almost covers all vital facets of disasters like disaster type, disaster location, disaster time, misfortunes including the causalities and the infrastructure loss, services, service providers, relief items, and so forth. The objectives of this research were to identify the requirements of a disaster ontology, to construct the ontology, and to evaluate the ontology developed for Disaster Trail Management. The ontology was assessed efficaciously via competency questions; externally by the domain experts and internally with the help of SPARQL queries.
\end{abstract}

Keywords-Semantic web; ontology; information retrieval; disaster trail management

\section{INTRODUCTION}

A large number of human beings are being affected by disasters every year. In disaster-affected areas, the survivors suffer a lot due to an interruption in the essential services like health care, communication, transportation, etc. Infrastructural damages can also affect food and water supply. Although the man has made considerable progress in the field of science, engineering, and technology, yet he is unable to control the occurrence of disasters. All his efforts, so far, aim at managing hazards, mitigation and to reduce the impact of disasters. Due to the devastating effects of disasters on human lives, catastrophes and crises management have always been given vital importance.

Disaster management is planning, arrangement, and deployment of resources with a precise aim of reducing disaster's damaging effects. Socio-economic conditions of the affected area and existence of effective information system regarding the occurrence of emergency are the significant factors that influence this management. Timely information plays a vital role in reducing disaster impact up to a certain level. The arrangements and organization of resources and efforts for mitigation majorly depend upon disastrous areas' situation and effects of the disaster on the local population. Efforts are made for gathering, organizing, and disseminating factual information to various stakeholders taking part in the mitigation process. Efficiency in the deployment of resources is one of the significant concerns in disaster management as it can minimize the disastrous aftereffects to a great extent.

\section{A. Overview}

The word 'disaster' itself shows that it is something troublesome that needs to be avoided or requires mitigation to bring down its outcomes if it ever happens again. Disaster mitigation focuses on long-term measures for diminishing risks. These measures can be structural or non-structural. Developing technological solutions and training of key personnel are examples of structural measures, whereas legislation and communicating potential threats to the public are considered as non-structural measures. Disaster mitigation or management process can be divided into three major phases:

- The data collection and analysis: Data is collected through observation techniques of the data collection and visualization, knowledge modeling, event forecasting, and information management after critical analysis.

- Data communication: Data communication or interconnectivity involves the mode of communication for information sharing among stakeholders.

- Data integration: Data integration phase involves combining the data from several disparate sources into meaningful and valuable information and providing a unified view to users. 
Traditional Information and Communication Technology (ICT) can contribute significantly to all the three phases discussed above. For collecting and compiling data with a view to its dispersal and assimilation, semantic web-based ICT solutions provide all these levels in a plausible way.

“The word 'disaster' comes from ancient Greek words dis means 'bad' and aster means 'star'. The astrological sense of disaster based on calamity blamed on star positions." (ewonago.wordpress.com)

Ontology is getting importance for providing clear and definite search by focusing the concepts in documents collection and data sources. Ontologies are designed to help improve communication, whether it is between human and machine or is between computers. In other words, ontology helps in managing knowledge.

An ontology primarily comprises of concepts (classes), properties (attributes), and possible relationships (slots) among concepts. There may exist some constraints (facets) on slots, or cardinalities on relationships among concepts. Collectively, the components and instances (individuals) form the knowledge base that helps in reasoning.

There are various types of ontologies which have been defined or discussed by multiple researchers, including:

- Upper ontology: very general concepts familiar to numerous domains for supporting the development of an ontology

- Domain ontology: domain-specific concepts

- Interface ontology: concepts relevant to the juncture of two disciplines

- Process ontology: knowledge domain of processes

Domain ontology is one of the classifications of ontology. Ontological design can better accumulate the knowledge of diverse nature as the disaster domain does. Hence, there is a need for a specific domain ontology to conceptualize the disaster trail management (DTM).

The primary purpose of designing ontologies is to formulate sketches for disaster plans. Operational centers set up for emergency may be aided with task ontology like study and planning of objects in disastrous scenes. Upper ontologies are enigmatic and abstract hence hamper understanding for those who are alien to its application. The developed DTM ontology covers nearly all sovereign states like nature of the hazard, occurrence date, damages like mislaying including the loss in infrastructure, refugee camps and facilities available or required in the refugee camp, rehabilitation tasks associated with the contributor, location, and relief index, and so forth.

Disasters cannot be avoided, but their effects can be minimized by active warning systems and better disaster trail management systems. In disaster management, real-time availability of information can improve the result-oriented rescue operations. Folk yield an enormous mass of information over blogs and social media that can be employed to aid relief services. A system can be devised to extract electronically the precise information related to disaster damages, filter, arrange, and format appropriately so that it can be utilized in disaster trail management. Consequently, semantic web technologies can perform a vigorous role in rendering up-to-date information that can later help disseminate to other stakeholders.

Disaster trail management is a challenging task due to its complexity and enormous requirements. "Insufficient coping capacity, pathetic communication, and collaboration between different concerned departments, lack of community awareness, resources gathering, and insufficient budgeting, lack of technology awareness, adoption and integration are the most common barriers in disaster management domain" [4].

Many efforts had been made in the field of ICT to breathe new life into dying humanity is itself not less than panacea in its benevolent magnitude. There are some ICT solutions available, but these solutions depend on hand-operated data entry. A massive amount of current data, shared on the internet, can be searched and utilized. Information from the internet can be retrieved via search engines, either keywordbased or semantic-based. Semantic-based search is more relevant to the user's information needs as it makes use of an ontology to get relations among query words to understand the meaning of words instead of searching only keywords and using page ranking algorithms which are the base of conventional keyword-based search. The machine-readable semantic features of the ontology result in the more contextual and significant search output.

Moreover, no domain ontology for disaster trail management has been developed and evaluated by earthquake-prone area domain experts for completeness and relevance by scrutinizing the competency questions. Some disaster-related ontology research work exists, but it does not serve the purpose of disaster trail management. To enhance the previous research work, and to overcome the highlighted issues, the development of disaster trail management ontology was needed to benefit the relevant users' group. This research will not only play its role to improve the ontology development in the disaster management domain but also add value to information retrieval in general. The research paper is divided into five sections. Remaining part of the article is organized in the following segments.

Section II reviews the existing ontologies for disaster management. Section III discusses the proposed disaster trail management ontology. Section IV deals with the ontology evaluation, and finally, the last part is dedicated to the conclusion and future work.

\section{LITERATURE REVIEW}

The research, as shows its title, carries some terms including ontology and disaster management; the literature review discusses both one by one. This section starts talking about previous ontology works. Then the discussion focuses on disaster-related ontologies and disaster management systems beginning with a brief description of disaster management phases; existing technological and semantic webbased solutions to disaster management. 


\section{A. Ontology: State of the Art}

Information Retrieval Systems not only have to deal with the structural complexity of complex databases but also with the semantic relationships between data which encourages the use of ontologies for knowledge representation [5]. Ontologies are growing more prevalent as a means for knowledge management, knowledge representation, knowledge sharing, and information retrieval, especially after the evolution of the Semantic Web technologies. An ontology represents a machine-understandable grammar consisting of concepts and relationships among these concepts to describe an area of knowledge [6, 7]. Among the causes of the increased prevalence of ontology is its capability to aid the information exchange between various systems, which is the significant success factor of the semantic web [8].

Many knowledge domains have successfully implemented and benefitted from ontologies for knowledge management including Genes analysis [9], chemical assessment framework [10], dairy industry [11], healthcare [12, 13], crises management [14], the music industry [15], agriculture [16], and so forth.

An ontological approach is establishing more practical in all developments of information retrieval, whether relating to opinion mining or cybercrime classification schemes. Practicing ontology with big data yields noteworthy gains in efficiency and productivity. Ontology finds extensive use in many domains, including machines learning, medical science, and genetic algorithms. Gruber [17, 18] defined ontology as "a formal, explicit specification of a shared conceptualization."

A need always drives every development; the same applies to ontology development as well. Following are some salient reasons that motivate researchers to develop an ontology.

- to share common domain knowledge

- to detach domain knowledge from the operational ones

- for a thorough analysis of domain knowledge to make it definite

- To qualify optimal reuse of domain knowledge in a specific area

\section{B. Disaster Management}

Due to the devastating effects of disasters on human beings, catastrophes and crises management have always been given vital importance. Disaster management is planning, arrangement, and deployment of resources with a precise aim of reducing disaster's damaging effects. Socio-economic conditions of the affected area and existence of an effective information system regarding the occurrence of emergency are the significant factors that influence this management. Timely information plays a vital role in reducing disaster impact up to a certain level. Efficiency in the deployment of resources is one of the significant concerns in disaster management as it can minimize the disastrous aftereffects.

Disaster management has four phases, namely, mitigation, preparedness, response, and recovery. Fig. 1 briefly describes the disaster management phases.

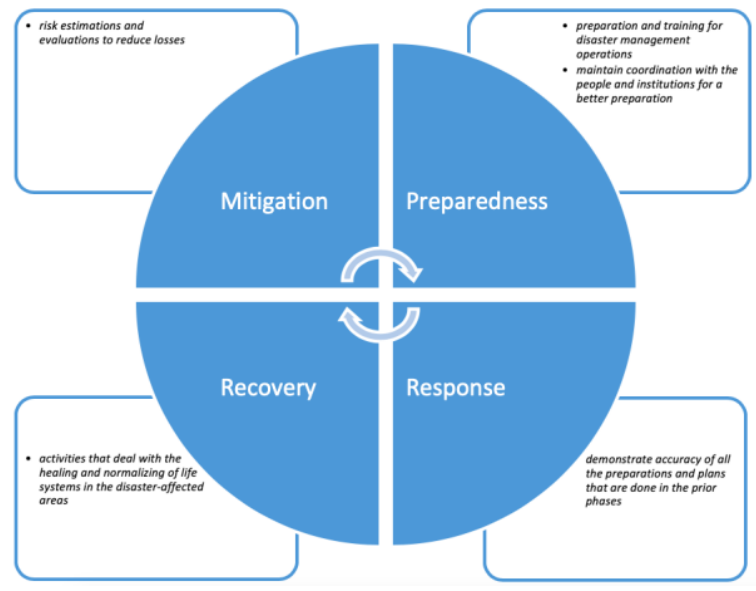

Fig. 1. The Disaster Management Cycle [19].

\section{Technological Advancements and Disaster Ontologies}

When disasters occur, government agencies, nongovernment organizations, and volunteers come forward for immediate rescue operations. First and foremost, the rescuers need to know about nature and disaster intensity, and the resulting damages and causalities. Secondly, the timeliness of information is critical. Disaster relief or control process executes three primary tasks, specifically data acquisition and interpretation, data communication, and data synthesis. Authoritative information and communication technology can offer notably to all the fundamental functions and expedite collaboration among workers and organizations. The evolution of ICT solutions has supported experts and researchers to devise such routines that operate more reasonably while incorporating all the necessary measures to alleviate and control the emergency. The research is improving present-day systems and is continuously making efforts to develop a perfect solution for disaster management.

The computer system deals with only those operations which run fluently without facing any hurdle. Disaster preparedness in computer science is a word depending wholly on a computer system, the central aim of which is data recovery. There is no much scientific contribution in improving the tools at hand and reshaping the ideas in this field. It must be the humanitarian duty of the scientists to study the problems related to the areas and affectees and provide prior research before callous mayhem. The research community has proposed many solutions, including semantic-based ontological solutions to address various needs of disaster management. Following are some worth noting semantic web-based contributions:

Kontopoulos et al. [14] represent an ontology for climate crises management. The proposed solution is claiming to cover all relevant aspects of the domain to facilitate a decision support system for crises management. The authors pointed out the overwhelming flow of varied information as the most critical challenge for decision making authorities and proposed a semantic ontological solution. The following set of three figures helps to understand the complete model of the proposed ontology. Fig. 2 elaborates natural disasters, Fig. 3 depicts analyzed data, whereas the Fig. 4 semantically represents the response unit assignments. 


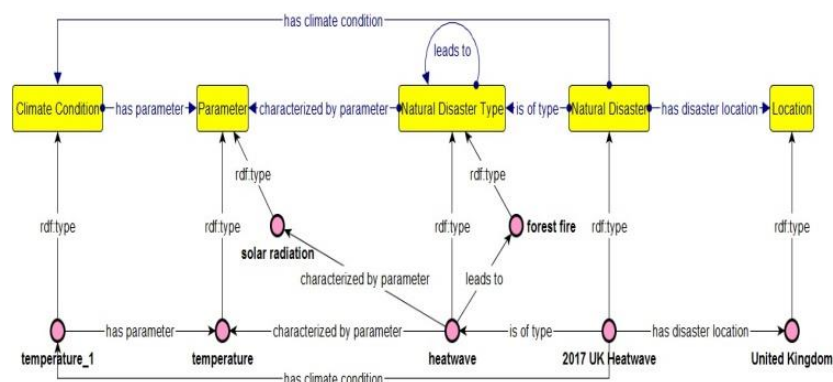

Fig. 2. Representation of Climate-Related Natural Disasters in the Proposed Ontology.

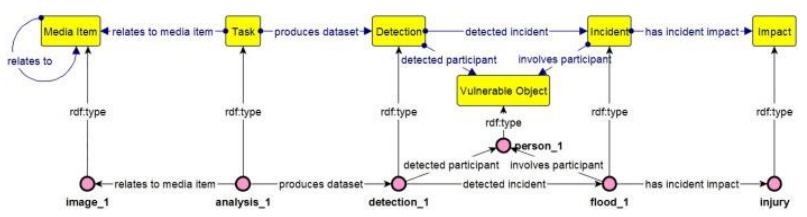

Fig. 3. Representation of Analyzed Data in the Proposed Ontology.

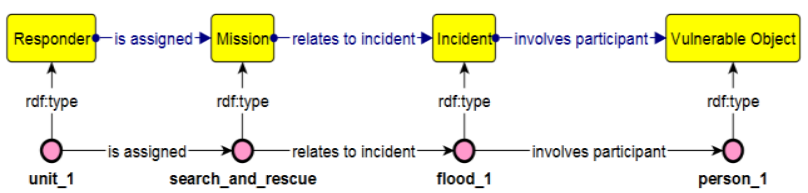

Fig. 4. Representation of Mission Assignments to First Responder units in the Proposed Ontology.

Notable Research Gaps: This research provides a knowledge base to help give authorities in decision support for emergency management. Although research challenges to include all the pertinent aspects of the domain, yet the focus of the study is on the response phase of disaster management. Thus, it has limitations to fulfill the requirements of the recovery phase like the orphan care, relief camps' services, facilities, and so on.

The study by Isbandono et al. [20] concentrates the capacity building of society through research, education, and training for handling disastrous situations. The study aims at disaster awareness in people by training in a planned and controlled manner along with handling media for information spreading regarding the emergency. The interest of the community to participate in disaster management activities can improve institutes' efficiency.

Notable Research Gaps: The research does not produce any concrete solution in the form of software or analytical methodology, instead, propose a program for developing awareness in society about crises to strengthen and provide support to the catastrophe management institutions by the participation of the community.

Bouyerbou et al. [21] proposed a geographic ontology to process satellite images after the disastrous event. Damages maps are prepared with the help of pre and post-disaster images by the photo-interpreter team, which is a complex and time-demanding task. The use of automatic or semi-automatic tools can make this activity easy and efficient. Automated processing has semantic limitations. This research proposed an ontological solution to reduce the semantic gap to help improve automatic processing and is evaluated with processing the Haiti 2010 satellite images. It consists of three sub-ontologies, namely surface, disaster, and damage. The surface sub-ontology illustrates geographical concepts. The concepts of disaster sub-ontology and damage sub-ontology are divided into two groups each - the former has Manmade and Natural, whereas the latter has Land Cover Damage and Material Damage groups. "The ontology aims to describe the content of satellite images, but a large number of concepts may cause complexity for an automatic process."

Notable Research Gaps: The ontology is aimed at the semantic annotation of satellite images but lacks in comprehensive domain representation, change detection, and detection of operational roads and the location of the highest priority areas like hospitals, residential buildings, and schools in the impacted areas.

Ahmad et al. [22] highlighted data problems of a disaster information management in setup and planning for emergency response and recovery stages and suggested improvements. They pointed out some challenges regarding data transmission that include data fragmentation, data transfer capacity of the correspondence channel, heterogeneity of the information structures. They described a data framework for system administration, apparatus arrangement, and resource reservation.

Peterson et al. [23] conducted a case study by engaging a team of 20 digital volunteers to capture medical-related information shared on social media after an earthquake disaster in Nepal. This study discusses potential strategies for future research joint ventures between the research and practitioner communities to utilize social media content. The research claims that near-real-time mission-specific actionable information can be generated during disasters and then used in decision-making.

Notable Research Gaps: The proposed system has some significant gaps. The data shared on social media has a lot of replication, and there should exist some mechanism to avoid duplications. The entire data floating on the public data stream is not factual; fake news or unauthentic information is also widespread, which need identification. Data shared in different forms, including text and multimedia. Only relevant data in an appropriate data type must be collected and analyzed. The data collection mechanism should be smart enough to accept only relevant data. Usage of abbreviation instead of complete words is widespread in messages on social media and needs to address correctly. Information shared in multiple languages, including native languages, require translation. Ignoring sharing in the local language may cause missing of essential data. The commonly used descriptive terms and area names need to be identified.

Zhou et al. [24] elaborated the model and faces of emergency decision making. In an emergency, decisions have to be taken in many areas of activity and at different levels; this increases complexity in forming a decision-makers group. Also, the natural difference in human perception, cognitive level, interest, and limited information can raise conflicts. Situation evaluation relies on experience and knowledge, which is not always enough in unexpected events. Also, the use of mathematical models and knowledge management tools 
in emergency decision making have their limitations. The study proposed a model for seismic infrastructure hazard by using Bayesian networks but lacks in the verification of the usefulness of the model in risk reduction.

In 2018, Inan et al. [25] proposed a decision support system for disaster management. The DSS mechanisms adopt an either bottom-up or top-down approach for decision making depending upon the knowledge trigger. Knowledge triggering factors maybe internal, i.e., the initiatives of disaster management authorities or may be external, i.e., the environmental changes. The study aims at specific disaster plans related to the volcanic eruption. A case study of Mt. Agung volcano eruption demonstrates the efficacy of proposed mechanisms. "The adopted knowledge analysis framework (KAF) allows the authorities to deal with uncertainties in the DM domain by understanding, analyzing, and finally structuring them into a format acceptable by the familiar stakeholders."

Notable Research Gaps: The authors feel that the system requires performance and efficacy evaluation in a real-time operational environment. They are also of the view that the DM agencies are lacking in fully documented DM plans. Although the research discusses the general scenario of disaster management domain yet, it focuses on addressing the disastrous situations caused by the volcanic eruption.

An effective early warning system for emergencies is a service to humanity because the intended population can take precautionary safety measures if they are aware of the crisis well in time. Moreira et al. [26] proposed an ontology-based EWS for alerting of distress. The study aims to develop an epidemiological surveillance EWS for the detection of infectious disease outbreaks in an area. Model-driven engineering framework of the system relies on the Situation Modeling Language (SML). The authors claim that their model is also suitable for the detection of floods, landslides, and wildfire.

Notable Research Gaps: The study proposed an ontologybased EWS for generating alerts to make the population aware of the imminent disaster. The focus of research is to detect infectious deceases outbreaks well in time although the authors are confident of their model to be suitable for detection of other catastrophes. The proposed model is not designed to work with post-disaster situations.

Anbarasi [27] proposed an ontology-based solution to crises management using data mining approaches. The study aims at developing a decision support system to address the information requirements of the disaster response. The software uses ontology concepts in the data mining framework. The authors named the ontology as a Humanitarian Assistance Ontology (HAO). Disaster-related data shared on social media can be collected for processing. Data integration and usage in the decision-making process in an emergency become difficult because of the data obtained from social media has structural and semantic heterogeneity. Fig. 5 illustrates the structure of HAO-system, whereas Fig. 6 portrays the architecture of this system.

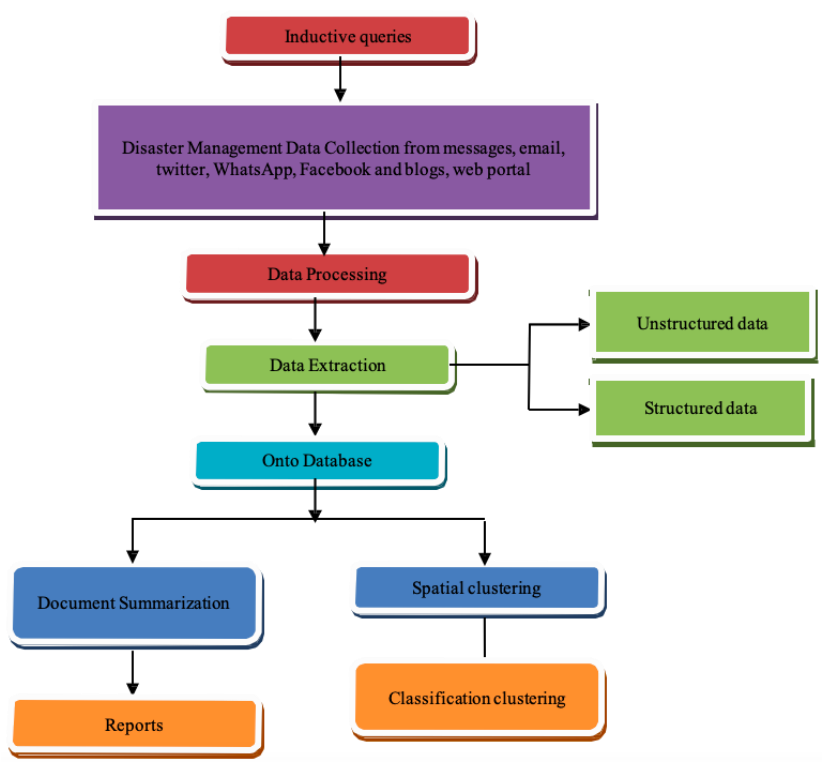

Fig. 5. Structure of HAO-System [27].

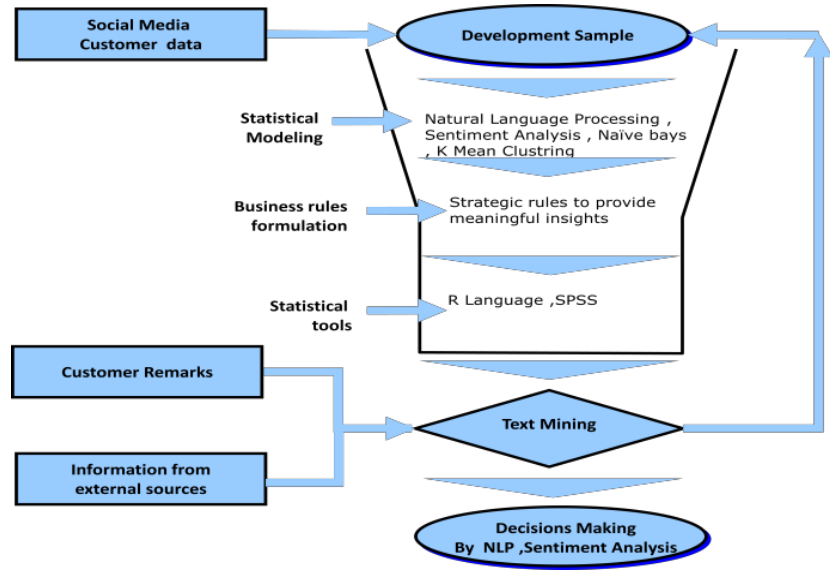

Fig. 6. Architecture of HAO-System [27].

Notable Research Gaps: The data acquisition from social media has some dilemmas and challenges which has been briefly discussed above in research gaps of [23]. The limitations of this research are quite similar to that.

Zhong et al. [28] presented a geo-ontology in 2016 as an emergency management solution for meteorological disasters. The factors of a meteorological disaster are typically time and space bound. Thus, the semantic relationships between concepts of the disaster domain are geographic locationspecific. Due to the geographic characteristics, the proposed solution to the meteorological disaster system is a GeoOntology. The primary objective of the ontology is to address the information needs of the preparedness and the response phases of meteorological disasters.

A geo-ontology based semantic conceptual model for earthquake emergency response knowledge was proposed by $\mathrm{Xu}$ et al. [29]. Geo-ontology can represent the geospatial aspects of the knowledge and satisfy the semantic needs of information interchange in the modeling process. "The model aims to solve knowledge problems to improve earthquake 
disaster response." The architecture classifies knowledge into four categories, namely factual knowledge, rule knowledge, procedural knowledge, and meta-knowledge. "The study presents a geo-ontology; and geo-ontology-based knowledge modeling primitives contain the spatial and earthly characteristics needed to adequately represent 'earthquakes on the ground,' rather than earthquakes in general."

Notable Research Gaps: The research focused on addressing response phase of disaster management and proposed a knowledge architecture only for earthquake disaster response.

Disaster management requires solutions for acquiring, analyzing, disseminating, and integrating data for which the information and communication technologies can play a viable role. Among several disaster management systems, Sahana has been exercised in numerous crises globally and recognized as the most mature DMS. Strenuous work of manual data entry is one of the vital issues of disaster management systems to date, which is quite a time consuming and tough, especially in case of crises. Immediate availability of essential information is always critical in crises management, which encourages to process the real-time data. Secondly, the existing DMS systems are semantically deficient, which is another significant problem to address.

A semantic web-based disaster management system is the solution to the issues highlighted, which is the best strategy to acquire, investigate, and share essential data where required. The semantic web uses ontologies as a fundamental constituent. Above discussions notified numerous ontologies, though all have limitations and expect structural reforms. Most of the research conducted so far aims at specific perspectives of the catastrophe administration domain.

\section{The Proposed DisAster TraIl MANAGEMENT ONTOLOGY}

There exist multiple ways to accomplish ontology development. In other words, there exists no exact and definite way or methodology for developing the ontologies. In the development of disaster trail management ontology, this research followed the methodology proposed by Noy \& McGuinness [1] in addition to taking guidance from the Protégé practical guide by Horridge et al. [30]. They proposed an iterative approach to ontology development by starting with a rough first pass at the ontology, revise and refine the evolving ontology and fill in the specifications. The research also uses a naming convention in ontology development to maintain uniformity and consistency of the ontology structure. The concept names start with a capital letter followed by lowercase letters, whereas the relationships and data properties begin with a lowercase letter. Relationship names are mostly of the form 'hasRelationship' and inverse relationship is of the type 'isRelationshipof'. Following steps have taken into consideration during the ontology development.

\section{A. The Domain and Scope of the Ontology}

Competency questions and their answers can be used as a tool to better understand the domain and scope of an ontology [31]. These questions are used as a litmus test for ontology because they determine whether the ontology contains enough information to answer this type of queries or not. This test also prompts whether the answers require a specific level of detail or representation of a particular area or not. The disaster trail management ontology should answer these competency questions. The list of prepared questions has been divided into three groups according to their nature, including Disasterrelated questions, Effects and Losses related questions, and Services and Facilities related questions. Although the competency questions are just a sketch and not the exhaustive list of inquiries, yet it includes the most appropriate questions which are enough to judge the domain and scope of the disaster trail management ontology. Following is a sample of a few questions from the list of competency questions.

- What is the magnitude and depth of the earthquake disaster?

- A secondary disaster is caused by which disaster?

- Floods are generated by bursting or seiche of which dam/water reservoir?

- How much area was damaged due to forest fire?

- How much is crops area affected by the disaster?

- What are relief items provided in the disaster area?

- What facilities are available in a refugee camp?

\section{B. Reusing Existing Ontologies}

It is admirable to see what others have achieved over time and to look forward to improving and enhancing the accomplishments for the specific domain. Reusing of existing ontologies may be essential in situations where the proposed ontology has to interact with other applications that have already been entangled with the controlled vocabularies [1]. This research has explored various ontology libraries but could not find any existing disaster ontology that answers the complete list of prepared competency questions. So, it was assumed that no relevant ontologies existed and started to develop the ontology from scratch.

\section{Enumeration of Important Terms in the Ontology}

It is a convenient approach to list down the comprehensive list of relevant terms of the concept, which are either required for making statements or need to clarify to a user. As the terms are the building blocks of an ontology, so, it is essential to be specific and clear about the principal terms and their related properties. This step addresses the basic concepts of the ontology.

For example, important earthquake disaster-related terms include, Earthquake, Disaster, name (disaster name), date (when the disaster occurred), magnitude, depth, Epicenter, Location (affected by the disaster), Activity (demanded), Damage, CalamityArea, Concomitant. There exist different relationships among these terms, including hasConsequence, hasEpicenter, hasAffect, hasDemand, and hasDamage. An earthquake may cause other disasters, secondary/concomitant disasters. For concomitant disaster-related terms may include the terms like Avalanche, Faulting, Fire, Flood, Landslide, RadioactivityFromNuclearPlant, Rockslide, SoilLiquefaction, spillOfChemicals, Tsunami, VolcanicEruption, 
areaTemperature, WaterReservoir and some relationships including isCausedByBurstOf, isCausedBySeicheOf, and isConsequenceOf, etc. (This is not an exhaustive list of defined terms).

\section{The Classes and the Class Hierarchy}

As far as the strategy of defining classes and class hierarchy is concerned, there are three types of approaches, namely top-down, bottom-up, and mixed designing approach. All three ontology design approaches are equally good. A developer can select any of these approaches depending on his/her personal view of the domain. According to Rosch [32], the combination design approach is the most convenient and practical approach for ontology development because the concepts "in the middle" tend to be more clear concepts in the domain.

Regardless of the adopted design approach, the development usually starts by defining classes. From the list of terms created in the "enumeration of important terms in the ontology" step, it is more convenient to start with selecting the terms describing the objects with independent existence. These terms become classes in ontology. After analyzing the disaster domain and having a critical view from the domain experts, the domain is conceptualized in the following way.

"Every OWL class is a subclass of Thing" (w3.org). Under the Thing class, the very first level of classes is considered as the top-level concepts in this documentation, such as Activity, CampFacility, Damage, Disaster, Location, Person, Miscellaneous, Organization, RefugeeCamp, ReliefItem, and Service are shown in Fig. 7.

Next level in the class hierarchical taxonomy is to define subclasses. For example, the concept of Activity is further divided into subclasses of StrategicPlanning and Vaccination. Among them, the concept, StrategicPlanning has specialized concepts of ResponseTeam, Rehabilitation, TaskReview, ScopeOfAction, and Evacuation. The hierarchical class taxonomy of Activity class is illustrated in Fig. 8.

The top-level concept of Damage is fractionated into subconcepts of Agriculture, Building, Crop, Forest, Infrastructure, and Livestock as illustrated in Fig. 9. Next level of subclasses is defined for Infrastructure which contains further specialized concepts of Airport, Bridge, CommunicationLine, FireStation, Road, ElectricitySupplyLine, FuelStation, GasSupplyLine, Seaport, MobileCommunicationTower, ParkOrPlayGround, RailwayTrack, SewagePipeLine, WaterReservoir, and WaterSupplyLine. Although this research did not capture all type of damages, it is tried to cover all those damages that are likely to occur.

\section{E. The Properties of Classes-Slots}

The properties of classes or the slots are defined to describe the internal structure of concepts as the defined terms of classes alone, do not contain enough information to answer the competency questions.

Some of the terms from the prepared enumerated list of important terms in the ontology are the concepts, called, classes and rest of the terms are the properties of the classes (slots) and facets to the slots. The remaining list of terms (except classes) is including the terms: address, adminName, age, animalHeads, animalType, area, areaTemperature, building, buildingType, byBurstHasCaused, bySeicheHasCaused, campNo, capacity, causeOfDeath, city, cnic, count, country, crop, damagedArea, damageEndPoint, damageStartPoint, damageType, date, dateOfDeath, deceased, depth, description, district, email, evacuated, fax, gender, handoverDate, hasAdopt, hasAffect, hasAreaUnit, hasBaseLocation, hasCalamityArea, hasCapacityUnit, hasCategory, hasConsequence, hasCurrency, hasDamage, hasDemand, hasEpicenter, hasExecute, hasFacility, hasGrant, hasLengthUnit, hasLocation, hasItem, heirs, injured, injury, isAdoptedBy, isAffectOf, isBaseLocationOf, isCalamityAreaOf, isCausedByBurstOf, isCausedBySeicheOf, isConsequenceOf, isDamageOf, isDemandOf, isEpicenterOf, isExecutionOf, isFacilityOf, isGrantOf, isLocationOf, item, itemType, lastClothing, lastSeenDate, lastSeenLocation, latitude, length, longitude, magnitude, missing, monetaryValue, name, noOfBeds, noOfBuildings, noOfRefugees, noOfStories, orgType, phone, quantity, regNo, reportedOn, state, supplyDate, tehsil, website, width, and zipCode.

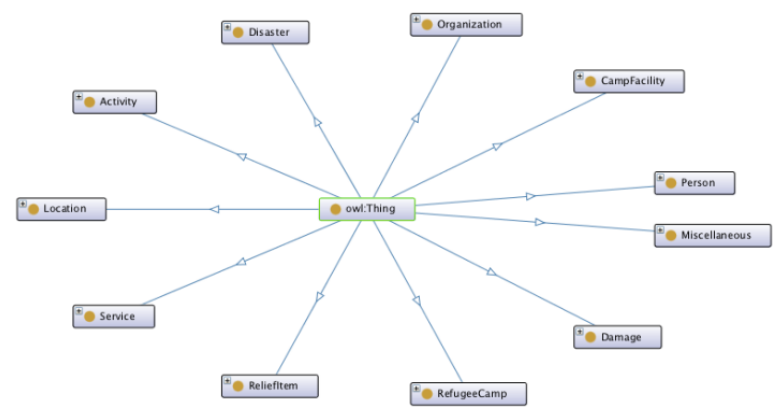

Fig. 7. Top-Level Concepts of DTM Ontology.

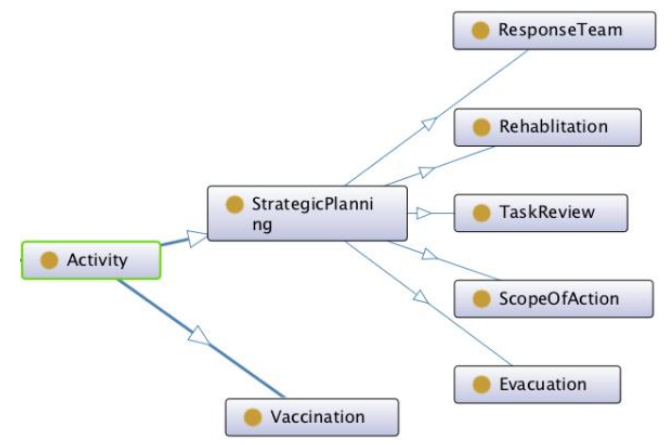

Fig. 8. Class Hierarchy of Activity Class.

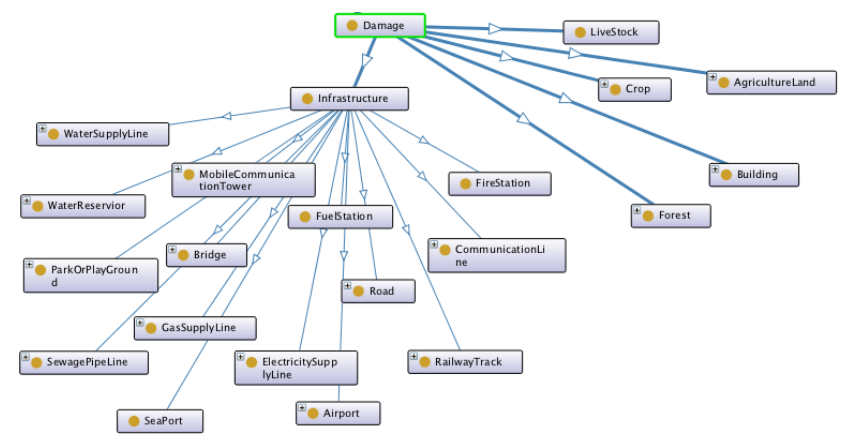

Fig. 9. Class Hierarchy of Damage Class. 
Now from the prepared list of properties of classes, it is required to determine which property is described for or related to which class. The properties of classes become slots attached to the classes.

For example, from the list of terms including Disaster, name, date, Location, Activity, CalamityArea, Damage, hasAffect, hasConsequence, hasDamage, and hasDemand, the terms hasAffect, hasConsequence, hasDamage, and hasDemand are the object properties because they associate the concept Disaster with some other concept. Whereas, the terms name and date are taken to be the data properties because they correlate the concept Disaster with some datatype value like string and dateTime, etc.

The properties defined in the above paragraph are assumed to be the essential part of every disaster; hence, are set at the root level. The focus of this study is on disaster trail management of earthquake, but an earthquake can cause other disasters as well. Thus, the concept Disaster has component classes of Earthquake and Concomitant (the concept to capture the knowledge of concomitant concepts of the earthquake).

In the class hierarchy, the properties of the parent class are inherited by its subclasses. Hence, the subsumed classes of Disaster, that is, Earthquake and Concomitant, inherit the properties from their subsuming class Disaster and also have additional properties defined at their level. For example, the Earthquake class adds the properties like hasConsequence (that associates Earthquake with its Concomitant disasters), hasEpicenter, depth, and magnitude, whereas, the concept Concomitant has the additional property isConsequenceOf, which is the inverse relationship of hasConsequence and correlates the concomitant disaster to its causing earthquake.

Concomitant is a generalized concept which is further fractionated into specialized concepts of disasters caused by the earthquake, like Avalanche, Faulting, Fire, Flood, Landslide, RadioactivityFromNuclearPlant, Rockslide, SoilLiquefaction, SpillOfChemical, Tsunami, and Volcanic Eruption. These inherited concepts share the properties defined in their parent concept Concomitant and grandparent concept Disaster along with their specific properties added at their level. For example, the concepts Avalanche, Faulting, Landslide, RadioactivityFrom NuclearPlant, Rockslide, SoilLiquefaction, SpillOfChemical, and VolcanicEruption also require the hasEpicenter property. Fire adds the properties of areaTemperature (data property), hasDamage (associate the Fire concept with Forest) and hasEpicenter. Along the same lines, the concept Flood needs additional properties of isCausedByBurstOf and isCausedBySeicheOf to associate this concept with another concept WaterReservoir. A volcanic eruption may also cause Tsunami; that's why the concept Tsunami requires an additional property hasConsequence to correlate this concept with its causing concept VolcanicEruption.

\section{F. The Facets of the Slots}

Facets are the restrictions on slots. A property restriction describes an anonymous class. All individuals that satisfy the restriction become a member of the anonymous class. A facet may represent the value type, value domain, cardinality, and other such feature related to a slot value. A slot may have different facets.

For example, the value of the slot name (as in "the name of a disaster") is a string, with a single value. That is, the name is a slot with value type string. A slot hasAffect (as in "a disaster has affected these locations") can have multiple values, which are instances of the class Location. That is, hasAffect is a slot with value type Instance with Location as an allowed class.

The value types used in the ontology are the integer, decimal, unsignedLong (for huge, whole numbers), string and dateTime. "Instance-type slots allow the definition of relationships between individuals. Slots with value type Instance must also define a list of allowed classes from which the instances can come [1]".

Let's start explaining the facets with the most prominent concept Disaster in the ontology. This concept is created with some other restrictions (anonymous classes), i.e., date exactly 1 dateTime, hasAffect some Location, hasConsequence some CalamityArea, hasDamage some Damage, hasDemand some Activity, name exactly 1 string, etc.

The restricted property hasAffect, is an object property whose domain is Disaster, the restriction type is some (existential) and the restriction filler is Location (the range of hasAffect). In other words, the restriction hasAffect some Location is an existential restriction (as denoted by the keyword, some), that works along with the hasAffect property, and has a filler Location. This restriction demonstrates the class of individuals that have at least one hasAffect relationship to an individual of the class Location. The restriction is a class which holds the individuals that satisfy the restriction. The restriction hasAffect is also depicted in Fig. 10, along with the other restrictions related to Disaster.

\section{G. The Hierarchical Taxonomy of Disaster}

Fig. 11 illustrates all the restrictions on the Disaster concept, which is the domain of all these restrictions. It is shown in the depiction that all object properties are existential whereas the datatype restrictions are exactly 1 . The restrictions hasConsequence, hasDamage and hasDemand have the concepts CalamityArea, Damage, and Activity as their respective ranges.

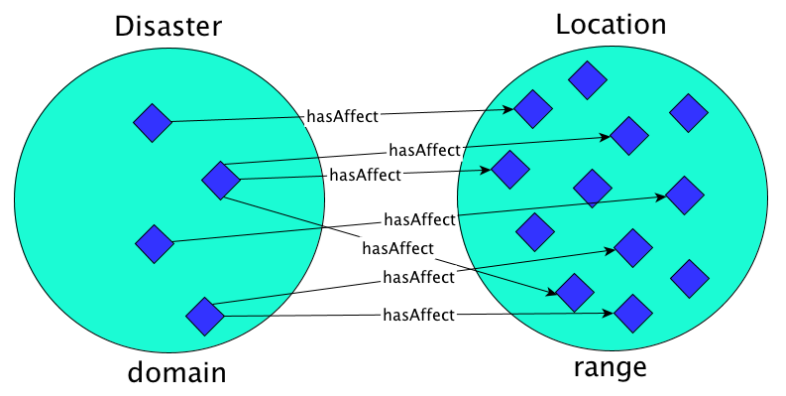

Fig. 10. HasAffect Object Property with Disaster as its Domain and Location as its Range (hasAffect Some Location). 


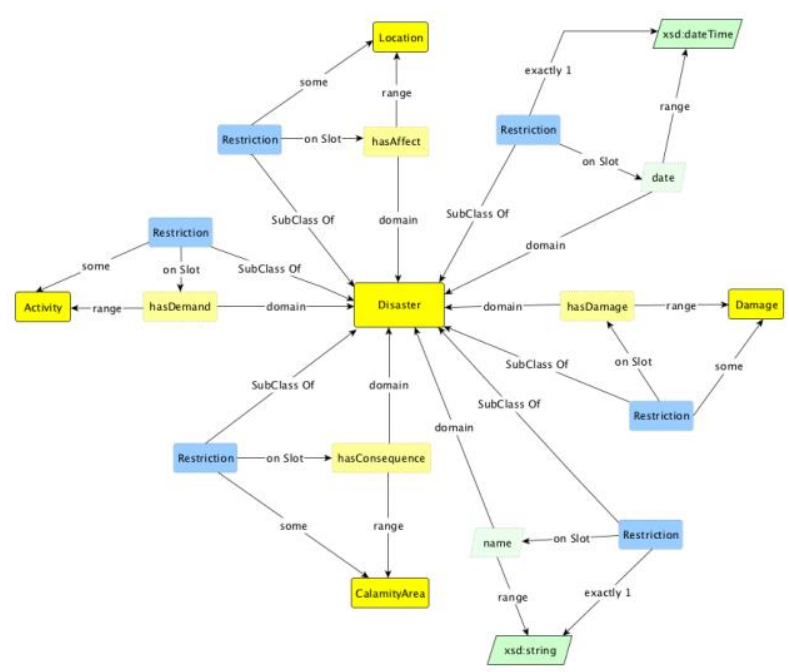

Fig. 11. The Concept Disaster with its Slots and Facets of the Slots.

The mathematical representation of the concept Disaster with its slots and facets is defined in Description Logic (DL) as follows.

Disaster $\subseteq \quad \exists$ hasAffect.Location $\cap$ $\exists$ hasConsequence.CalamityArea $\cap$ ᄏhasDamage.Damage $\cap$ $\exists$ hasDemand.Activity $\cap \quad \mid \exists$ date.dateTime $\mid=1 \quad \cap$ $\mid \exists$ name.string $\mid=1$

The concept Disaster has two inherited concepts Earthquake and Concomitant. The following figure depicts the slots and facets of slots of subsumed concept Earthquake. The concept Earthquake, has two additional object properties, namely hasConsequence and hasEpicenter (along with the inherited properties from subsuming concept Disaster), as illustrated in the following figure. The slot, hasConsequence some Concomitant, as shown in the representation, has restriction type existential (some) and the slot is linked with the concept Earthquake as the domain of the slot and the concept Concomitant as the range. Another slot, hasEpicenter exactly 1 Epicenter, has exact cardinality equal to 1 and attached to the concept Epicenter as the range of the slot. Earthquake is defined with two additional datatype properties, namely depth, and magnitude. Both the slots have existential facets and are linked to decimal datatype as their range.

The mathematical representation in DL, of the concept Earthquake, as portrayed in Fig. 12, can be defined as follows.

\section{Earthquake $\subseteq$ Disaster $\cap \exists$ hasConsequence.Concomitant $\cap \mid \exists$ hasEpicenter.Epicenter $\mid=1 \cap \exists$ depth.decimal $\cap$ $\exists$ magnitude.decimal}

Another inherited concept of Disaster, the Concomitant, with all its additional slots and inherited concepts are described in Fig. 13. For simplicity of the representation, the subsumed concepts of Concomitant shown in the bluebordered rectangle are not shown with their slots and is portrayed in Fig. 14. Concomitant has one additional existential object property is consequence of some Earthquake which is an inverse object property of the concept Earthquake (has consequence some Concomitant, presented in Fig. 12).

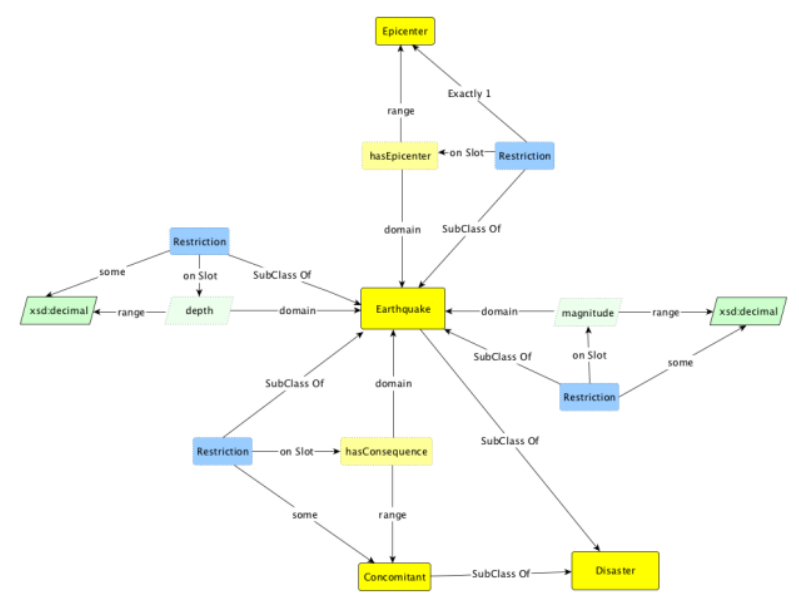

Fig. 12. The Concept Earthquake with its Slots and Facets of the Slots.

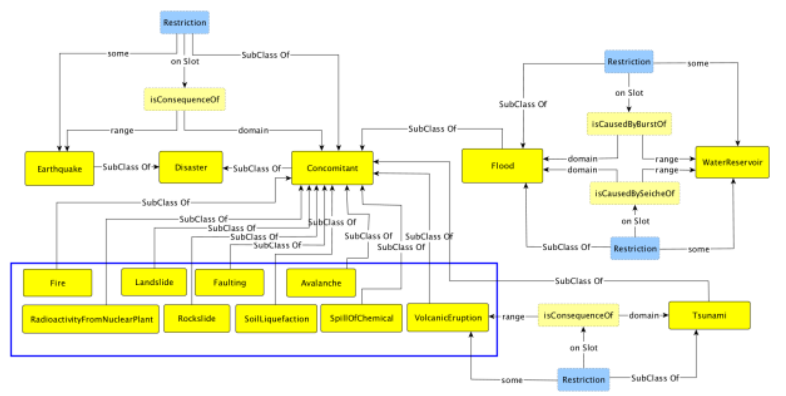

Fig. 13. The Concept Concomitant with its Slots and Facets of the Slots.

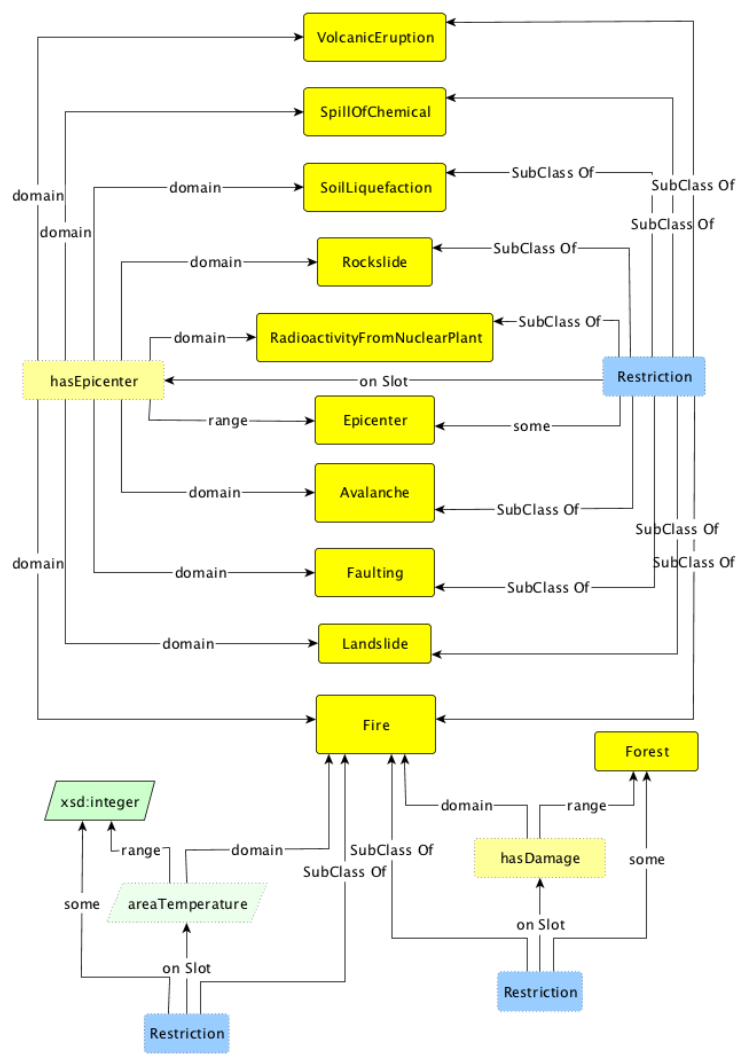

Fig. 14. The Subpart of the Concept Concomitant with its Slots and Facets of the Slots 
The inherited concept Flood of Concomitant has two additional existential object properties; isCausedByBurstOf some WaterReservoir and isCausedBySeicheOf some WaterReservoir as presented by the following figure. Two subclasses of Concomitant, Tsunami, and VolcanicEruption are linked to each other via an existential slot isConsequenceOf having Tsunami as the domain and VolcanicEruption as the range.

Avalanche, Faulting, Fire, SoilLiquefaction, Landslide, Rockslide, RadioactivityFromNuclearPlant, SpillOfChemical, and VolcanicEruption are the inherited concepts of Concomitant, all having an addition slot hasEpicenter some Epicenter which is an object property of existential restriction type and is illustrated by the Fig. 14. The concept Fire has another addition existential object property hasDamage some Forest and a datatype property areaTemperature some integer which also has an existential facet.

Mathematical representations in DL, of all the concepts presented in the above Fig. 13 and 14 are as follows.

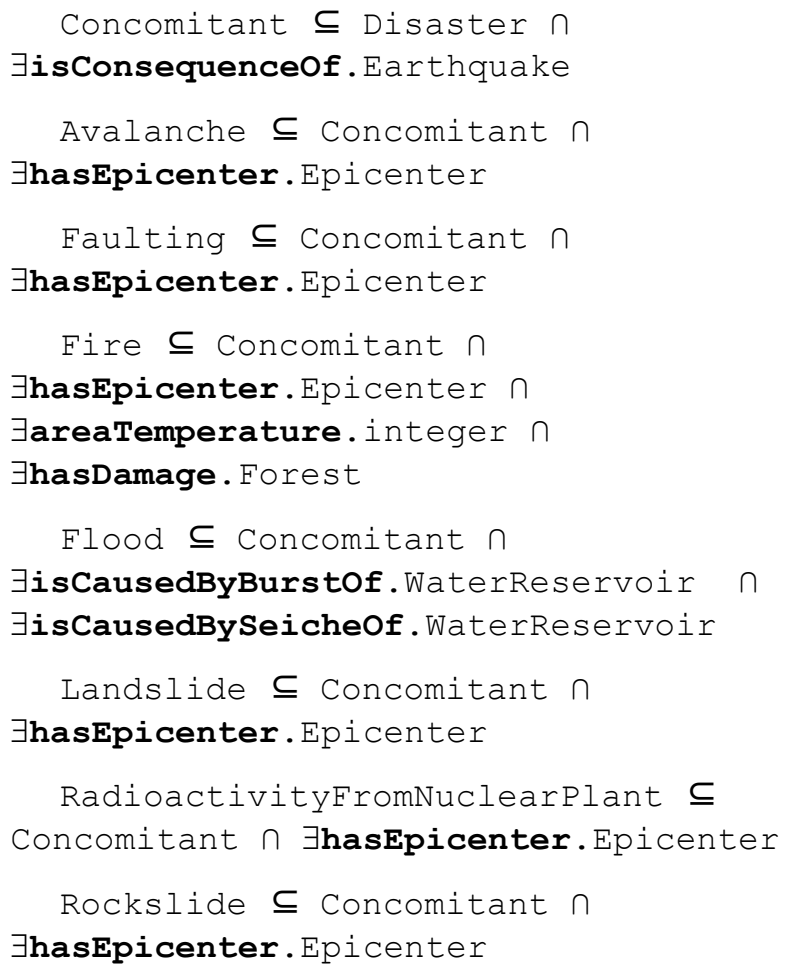

\section{ONTOLOGY EVALUATION}

Pinto \& Martins [33] expressed ontology evaluation as a technical process of judging the quality of the ontology. Various ontology evaluation techniques are found in past research work. The ontology evaluation of this research follows the guidelines and criteria recommended by Gomez et al. [2] and Kreider [3]. Ontology evaluation is essential to promise the overall quality of the developed ontology. It not only assesses the technical aspect of the ontology but also encourages the domain experts' involvement for their worthy judgment. The efficacy of ontological knowledge depends on the quality of the ontology, and evaluation is a way to gauge the level of quality of the ontology.

This section presents some ontology evaluation techniques accompanied by significant ontology evaluation results. The results produced by the process of assessment delineates the correctness and usefulness of the ontology. The term "Evaluation" subsumes the terms "Validation" and "Verification". The validation process is completed by involving domain experts, whereas, for the verification process, the use of a software technique is brought into work. As a first step and to measure the domain and scope of ontology, some appropriate competency questions (CQs) are fabricated, which are then evaluated by the domain experts. The relevance and completeness of these CQs validate the ontology. Verification process gauges the correctness and usefulness of the ontology, which is done by developing queries in SPARQL to provide solutions to the CQs.

According to Fernández et al. [34], verification speaks about the activity that assures the correctness of an ontology. Vrandečić [35] refers to verification as an evaluation task for assessing that the ontology has been built correctly. This task can be performed during each phase of ontology development or between phases of the development life cycle. This technical process of verification guarantees the usefulness and accuracy of an ontology according to the accepted understanding of the domain of specialized knowledge sources. The verification process can be performed by technically generating answers to the CQs using some appropriate query language. This research is using SPARQL query language.

The methodology of ontology evaluation by generating answers to CQs using a query language is adopted by many researchers.

This section furnishes the results produced from the execution of CQs codified in SPARQL, a query language. CQs are among the foremost applied and familiar context for ontology assessment. This competency appraisal is performed to examine the preciseness of the ontology using query language in the ontology development tools, for example, DL queries or SPARQL queries. SPARQL query is the commonly used plugin within Protégé. The SPARQL Query is used as a tool to gauge the adequacy of the ontology. In the frame of reference for this research, SPARQL queries will be developed for the execution of CQs. CQs are composed at the ontology specification stage to define the scope of the ontology. The concluded set of CQs are then codified in the query language before the execution on SPARQL Query. 
"SPARQL allows for a query that consists of triple patterns, conjunctions, disjunctions, and optional patterns. SPARQL does not have a native inference mechanism incorporated into the language. SPARQL queries return what is contained in the information model in the form of graph bindings [36]". To get some appropriate results from a SPARQL query, the individuals can be added to the ontology with some actual or sample data. These queries will help evaluate the ontology and may also help to improve the architecture of the ontology. The CQs are listed in section III (A). The representation is in three folds starting with natural language query (question) followed by the SPARQL query and finally the result produced by the execution of SPARQL query.

Following PREFIXs are used in all SPARQL queries

PREFIX rdf:

<http://wWw.w3.org/1999/02/22-rdf-syntaxns\#>

PREFIX OWI:

<http://www.w3.org/2002/07/owl\#>

PREFIX rdfS:

<http://www.w3.org/2000/01/rdf-schema\#>

PREFIX XSd:

<http://www.w3.org/2001/XMLSchema\#>

PREFIX dtm:

<file://ashfaqahmad/DTM.owl\#>

CQs:

3. When and where the earthquake disaster occurred?

4. What is the magnitude and depth of the earthquake disaster?

5. What are the latitude and longitude of the earthquake Epicenter?

SPARQL Query:

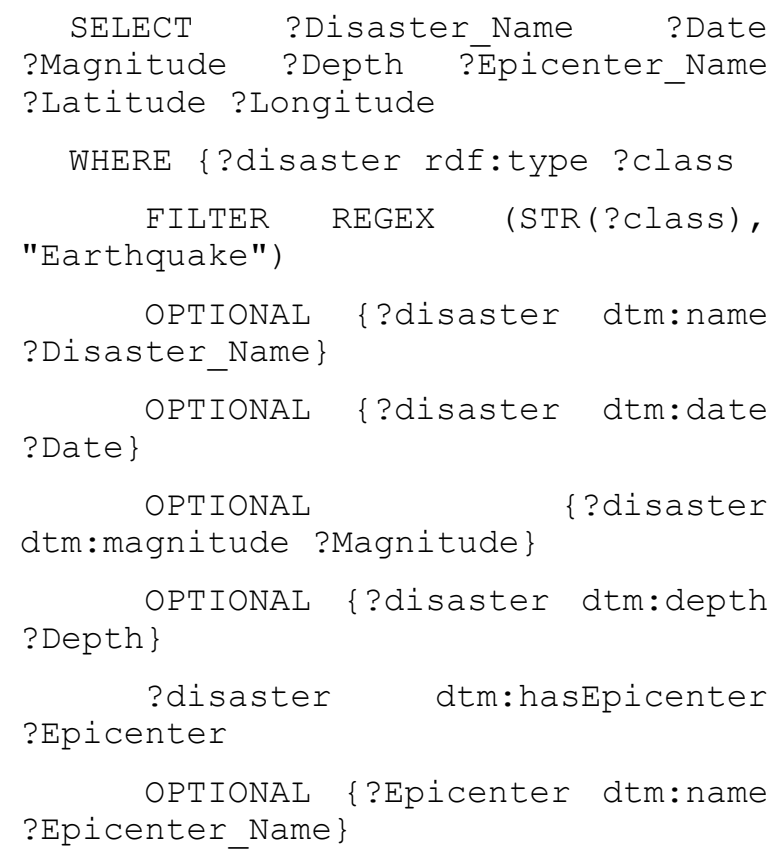

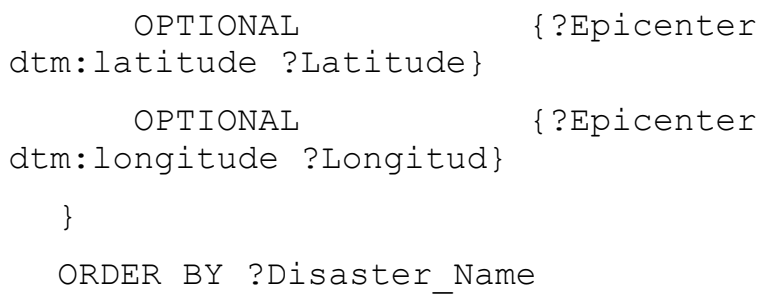

$\{$ ? Epicenter

$\{$ ? Epicenter wn Fig. 15.

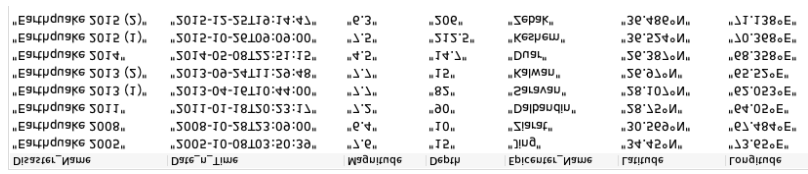

Fig. 15. Results of SPARQL Query for CQs 1-3.

CQ 4: A secondary disaster is caused by which disaster?

SPARQL Query:

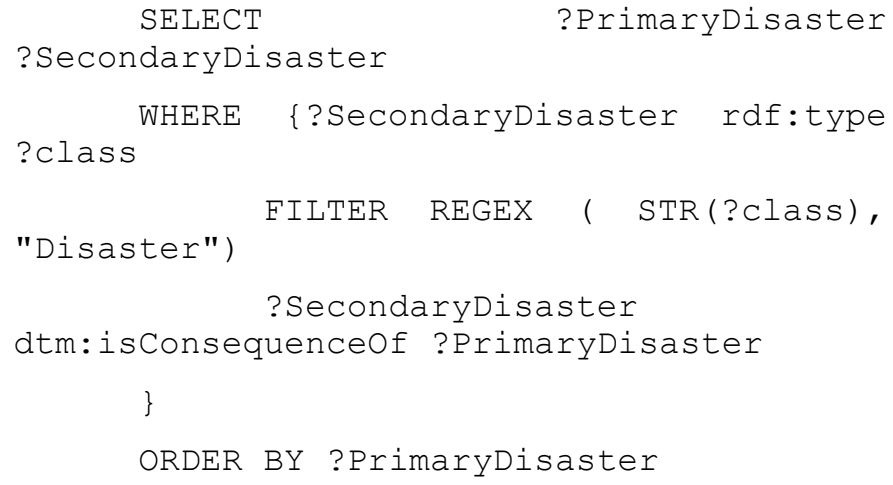

CQ 5: Floods are generated by bursting or seiche of which dam/water reservoir?

SPARQL Query:

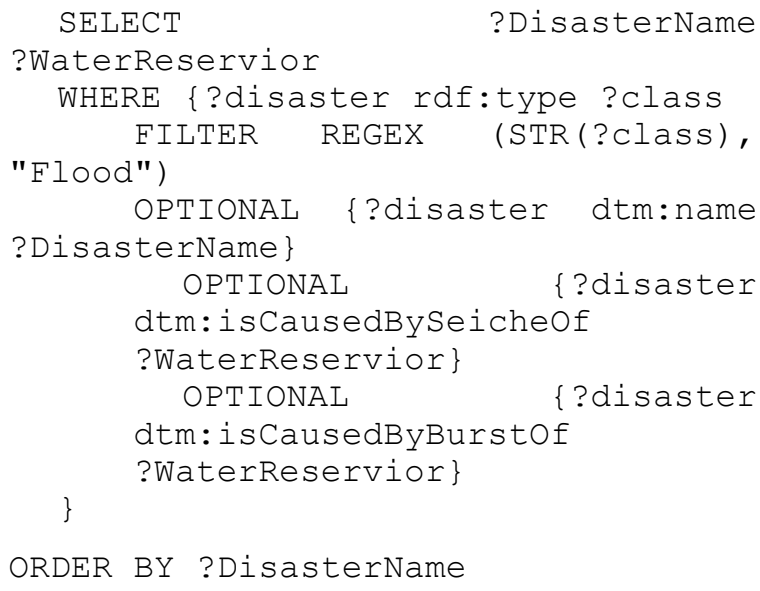

6. What is the total area of the forest, under fire?

7. How much area was damaged due to forest fire?

8. What was the area temperature (degree Celsius) during the fire?

SPARQL Query: 
SELECT ?DisasterName ?ForestName ?DamagedArea ?TotalForestArea ?AreaUnit

?AreaTemperatureDuringFire_DegreeC ?EpicenterName

WHERE \{

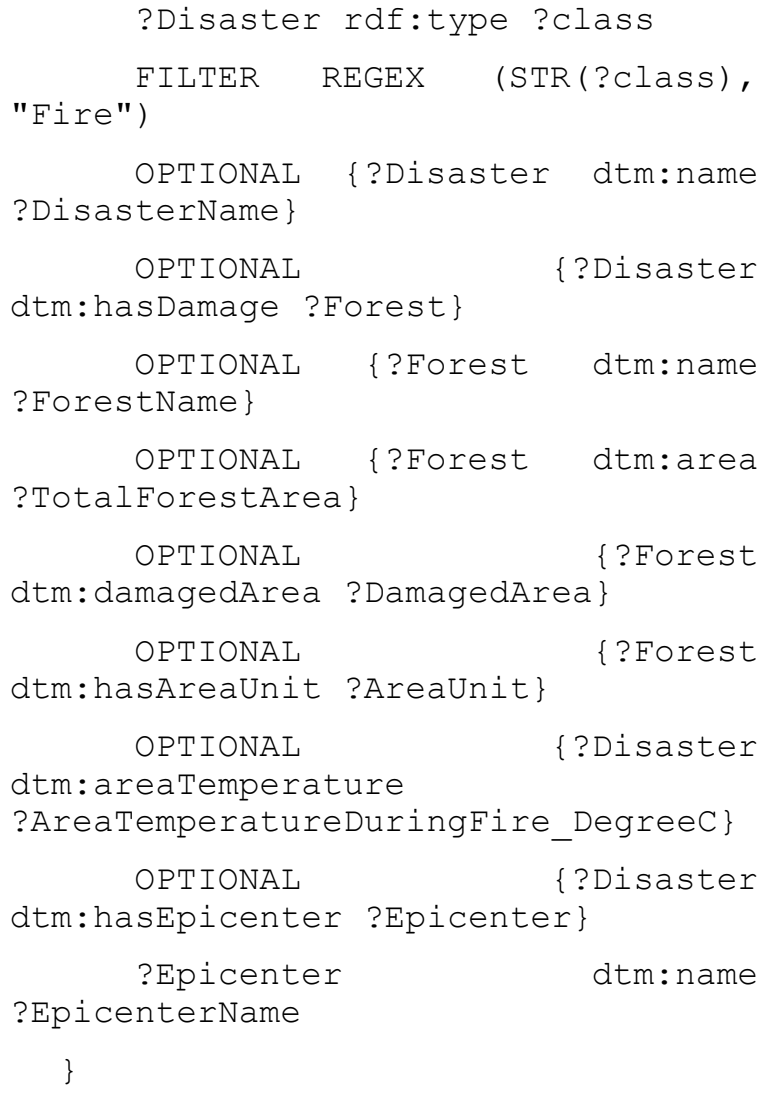

CQ 9: How much crops area is affected by the disaster?

\section{SPARQL Query:}

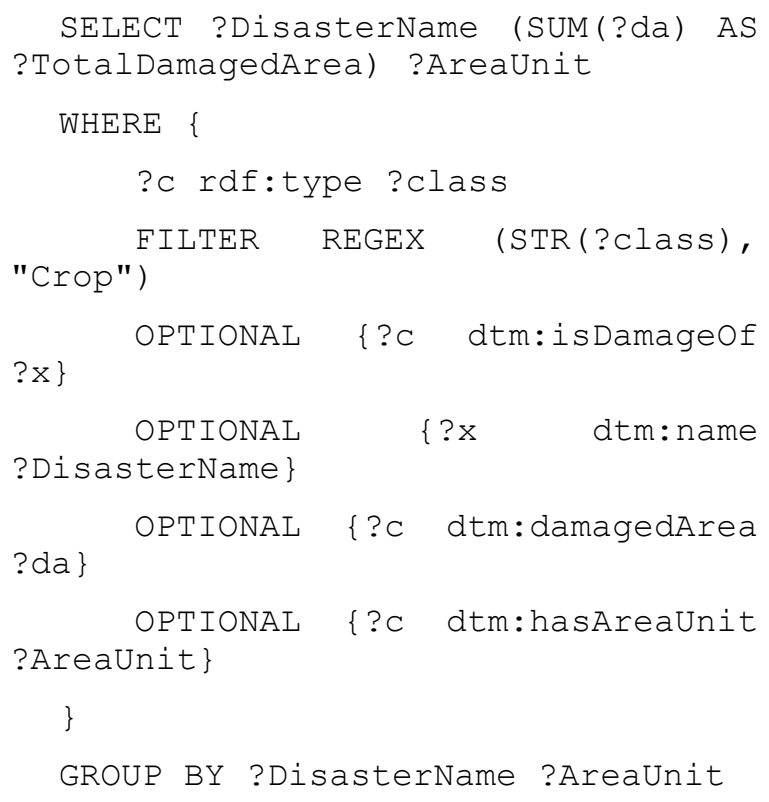

CQ 10: What relief items are provided in the disaster area?

\section{SPARQL Query:}

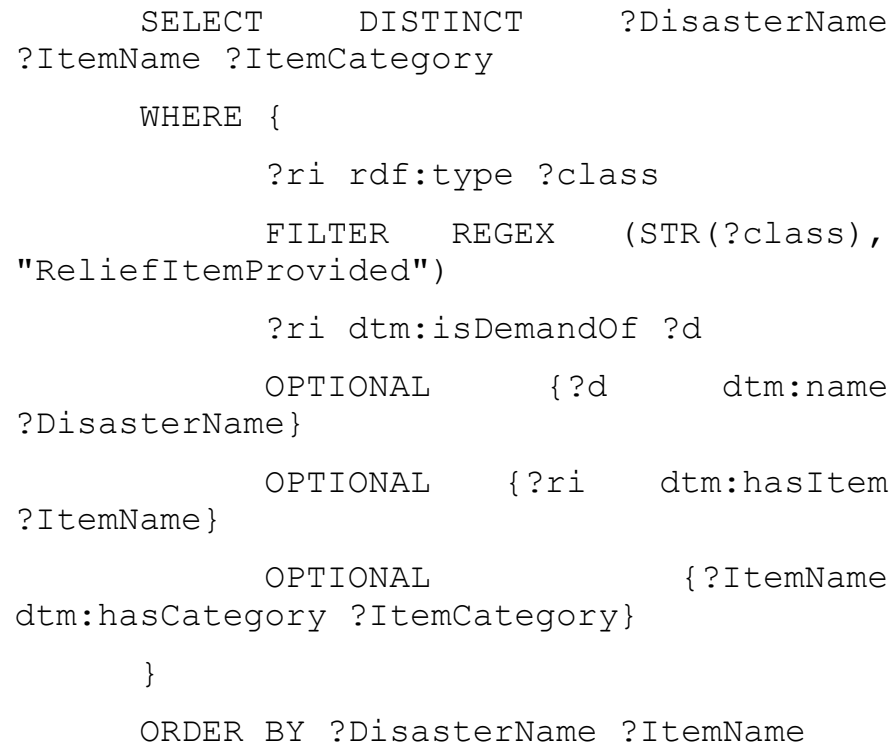

CQ 11: What facilities are available in a refugee camp? SPARQL Query:

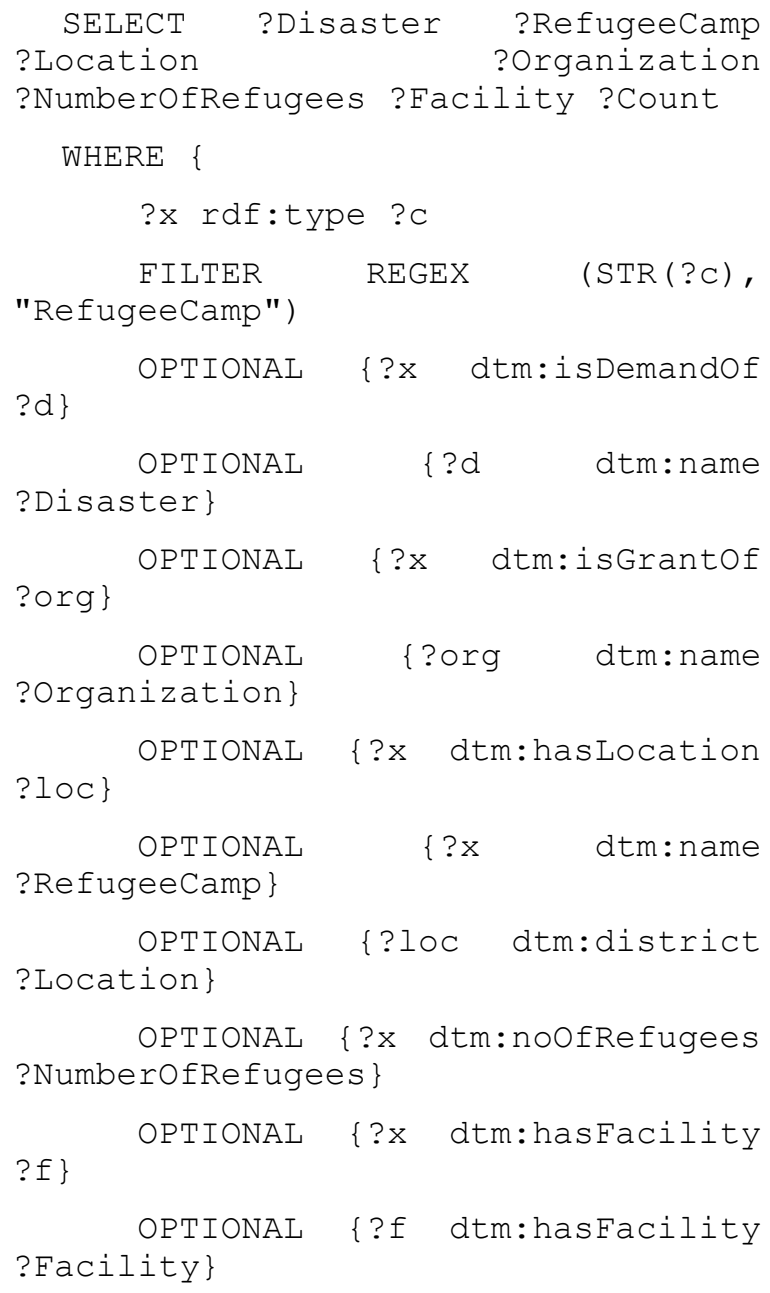




\section{OPTIONAL ?Count \\ $\{$ ? $\quad$ dtm:count \} \\ ORDER BY ?Disaster ?Location ?Facility}

\section{CONCLUSION AND FUTURE WORK}

On the first hand, the ontology designing is an innovative development. Thus, different ontology developers would undoubtedly come up with ontologies designed for different purposes. Secondly, multiple ontologies may serve the object for a domain correctly. This research proposes an ontology for disaster trail management. The study included the relevant concepts very carefully after analyzing the real data from credible sources, disaster-related news, and discussion of the scenario with domain experts. Positive comments by the domain experts on ontology validation assessment and generated results of SPARQL queries executed for relevant CQs showed that the ontology meets the required criteria. The research proposed a distinct and cardinal ontology which encompasses the entire domain of earthquake disaster trail management for Pakistan using appropriate semantic relationships among the ontology concepts. It is hoped that the semantic web research community will contribute further to enhance the ontology to make it fit for all type of disasters including human-made disasters and for accommodating region-specific requirements of other countries in the world.

Whether we talk about ontology development or an architectural model for an application, these are completed through a progressive approach. Thus, no such work can be assumed as ultimate, and there is always room for modification and enhancement. Following are the recommendations by this study as future work to enhance the proposed semantic web-based ontology.

- The proposed ontology can be improved to a multilingual corpus.

- The ontology aims at addressing the disaster trail management in Pakistan, which can be modified or enhanced to fit the region-specific requirements of other countries.

- The ontology design can be improved to accommodate human-made disasters like war.

\section{REFERENCES}

[1] Noy, N. F., \& McGuinness, D. L., "Ontology development 101: A guide to creating your first ontology," 2001.

[2] Gómez-Pérez, A., Fernández-Lopez, M., \& Corcho, O., "Ontological Engineering: with examples from the areas of knowledge management, ecommerce and the Semantic Web," Book by Springer, 2004.

[3] Kreider, R. G., "An ontology of the uses of building information modeling," 2013.

[4] Shafiq, F., \& Ahsan, K., "An ICT based early warning system for flood disasters in Pakistan,” Res. J. Recent Sci. ISSN, 2277, 2502, 2014.

[5] Munir, K., \& Anjum, M. S., "The use of ontologies for effective knowledge modelling and information retrieval," Applied Computing and Informatics, 14(2), 116-126, 2018.

[6] Kapoor, B., \& Sharma, S., "A comparative study ontology building tools for semantic web applications," International Journal of Web \& Semantic Technology (IJWesT), 1(3), 1-13, 2010.
[7] Ranpara, R., Yusufzai, A., \& Kumbharana, C. K., "A Comparative Study of Ontology Building Tools for Contextual Information Retrieval," In Emerging Trends in Expert Applications and Security (pp. 401-408), Springer, Singapore, 2019.

[8] Noy, N. F., "Semantic integration: a survey of ontology-based approaches," ACM Sigmod Record, 33(4), 65-70, 2004.

[9] Liu, S. Q., Cheng, J. Y., Jiang, Y. J., Li, Y., Li, Q. H., \& Pang, T. X., "Bioinformatics Analysis and Verification of Aging-Related Genes of Bone Marrow Mesenchymal Stem Cells in Patients with Acute Myeloid Leukemia. Zhongguo shi yan xue ye xue za zhi," 27(2), 311-317, 2019.

[10] Ouya, S., "Chemical Assessment Framework and Ontology," In Mobile Technologies and Applications for the Internet of Things:, Proceedings of the 12th IMCL Conference (p. 385), Springer, 2019.

[11] Sun, Y., Wang, C., Sun, X., \& Guo, M., "Comparative Proteomics of Whey and Milk Fat Globule Membrane Proteins of Guanzhong Goat and Holstein Cow Mature Milk," Journal of food science, 84(2), 244-253, 2019.

[12] Cahyani, D. E., Prabanuadhi, A., Irfan, R. I., \& Febrianto, L. A., "Ontology Model for Dietary of Children with Autism Spectrum Disorders," Journal of Telecommunication, Electronic and Computer Engineering (JTEC), 10(2-4), 129-132, 2018.

[13] Afandi, R. R., Radman, A., Bahari, M., Zakaria, L. Q., Mustapha, M., \& Ismail, W., "Ontology Development in Patients Information System for Stroke Rehabilitation," In CEUR Workshop Proceedings (Vol. 2137), January 2017.

[14] Kontopoulos, E. Mitzias, P.Moßgraber, J.Hertweck, P.van der Schaaf, H.Hilbring, D.Lombardo, F.Norbiato, D.Ferri, M.Karakostas, A.Vrochidis, S.and Kompatsiaris, I., "Ontology-based Representation of Crisis Management Procedures for Climate Events," 1st Int. Workshop on Intelligent Crisis Management Technologies for climate events (ICMT), 2018.

[15] $\mathrm{Xu}, \mathrm{H} .$, "Whether the Work Type Debates in Higher-Level Musical Ontology Are Useful Just for Philosophy Itself?," 2018.

[16] Deb, C. K., Karn, S. K., Das, M., \& Marwaha, S., "Microbial Taxonomy Ontology for Agriculturally Important Microorganisms (AMO) Coupled with Sequence Alignment Reinforcement Options," Int. J. Curr. Microbiol. App. Sci, 7(4), 3154-3166, 2018.

[17] Gruber, T. R., "A Translation Approach to Portable Ontology Specifications," Knowledge Acquisition 5(2), 199-220, 1993a.

[18] Gruber, T. R., "Toward Principles for the Design of Ontologies Used for Knowledge Sharing," International Journal of human-computer studies, 43(5), 907-928, 1993b.

[19] Ahmad, A., Othman, R., \& Fauzan, M., "A Social Semantic Web Based Conceptual Architecture of Disaster Trail Management System," International Journal of Advanced Computer Science and Applications (IJACSA), 8(4), http://dx.doi.org/10.14569/IJACSA.2017.080437, 2017.

[20] Isbandono, P., Prastyawan, A., \& Gamaputra, G., "The capacity building of disaster management in Bojonegoro regency," In Journal of Physics: Conference Series (Vol. 953, No. 1, p. 012162). IOP Publishing, January 2018.

[21] Bouyerbou, H., Bechkoum, K., \& Lepage, R., "Geographic ontology for major disasters: Methodology and implementation," International Journal of Disaster Risk Reduction, 2018.

[22] Ahmad, S., Rahman, I., Mallik, A., \& Arefin, A., "Integrated Information System for Disaster Management: A Creative Case Study," International Journal of Information, Business and Management, 10(2), 64-76, 2018.

[23] Peterson, S., Thompson, C., \& Graham, C., "Getting Disaster Data Right: A Call for Real-time Research in Disaster," 15th International Conference on Information Systems for Crisis Response and Management (ISCRAM), May 2018.

[24] Zhou, L., Wu, X., Xu, Z., \& Fujita, H., "Emergency decision making for natural disasters: An overview," International journal of disaster risk reduction, 27, 567-576, 2018.

[25] Inan, D. I., Beydoun, G., \& Pradhan, B., "Developing a decision support system for Disaster Management: Case study of an Indonesia volcano eruption," International journal of disaster risk reduction, 31, 711-721, 2018. 
[26] Moreira, J. L., Pires, L. F., van Sinderen, M., \& Costa, P. D., "OntologyDriven Conceptual Modeling for Early Warning Systems: Redesigning the Situation Modeling Language," In MODELSWARD (pp. 467-477), February 2017.

[27] Anbarasi, C., Mayilvahanan, P., \& Pallavaram, C., "Humanitarian Assistance Ontology Implementation during Disaster Management in Chennai Flood-2015 Using Text Mining Techniques,” International Journal of Pure and Applied Mathematics, 116(21), 729-739, 2017.

[28] Zhong, S. B., Wang, C. L., Yao, G. N., \& Huang, Q. Y., "Emergency Decision of Meteorological Disasters: a Geo-Ontology Based Perspective," DEStech Transactions on Computer Science and Engineering, (cmsam), 2016.

[29] Xu, J., Nyerges, T. L., \& Nie, G., "Modeling and representation for earthquake emergency response knowledge: perspective for working with geo-ontology," International Journal of Geographical Information Science, 28(1), 185-205, 2014.
[30] Horridge, M., Jupp, S., Moulton, G., Rector, A., Stevens, R., \& Wroe, C., "A practical guide to building owl ontologies using protégé 4 and coode tools edition1.2," The University of Manchester, 107, 2009.

[31] Grüninger, M., \& Fox, M. S., "Methodology for the design and evaluation of ontologies," IJCAI-95 Workshop on Basic Ontological Issues in Knowledge Sharing, Montreal, 1995.

[32] Rosch, E., "Principles of categorization," Cognition and categorization, ed. by Eleanor Rosch \& Barbara B. Lloyd, 27-48, 1978.

[33] Pinto, H. S., \& Martins, J. P., "Ontologies: How can they be built?," Knowledge and information systems, 6(4), 441-464, 2004.

[34] Fernández, M., Gómez-Pérez, A. \& Juristo, N., "METHONTOLOGY: From Ontological Art Towards Ontological Engineering," AAAI-97 Spring Symposium on Ontological Engineering, Stanford University, March 24-26 ${ }^{\text {th }}, 1997$.

[35] Vrandečić, D., "Ontology evaluation," In Handbook on ontologies, Springer, Berlin, Heidelberg, 2009, pp. 293-313.

[36] Pedro, V.C., Nyberg, E., and Carbonell, J., Federated ontology search (Doctoral dissertation, Carnegie Mellon University, Language Technologies Institute, School of Computer Science), 2009. 